\title{
Effect of smokeless tobacco use on salivary glutathione levels among chronic periodontitis patients before and after non- surgical periodontal therapy
}

\author{
Arati C. Koregol', Nagaraj B. Kalburgi', Pushpa Pattanashetty', Shivaraj Warad', Nandini S. Shirigeri', Vani C. Hunasikatti'
}

\begin{abstract}
INTRODUCTION Smokeless tobacco (SLT) jeopardizes periodontal health and also produces an imbalance between reactive oxygen species (ROS) and antioxidants (AO) such as glutathione. Glutathione is an important redox regulator in saliva and its maintenance is essential for periodontal health. Periodontitis patients have a reduced total $\mathrm{AO}$ capacity in whole saliva, and periodontal therapy restores the redox balance. Hence, the purpose of this study was to investigate the effects of smokeless tobacco use on saliva glutathione levels in patients with chronic periodontitis and to evaluate these effects after non-surgical periodontal therapy. METHODS The study included 100 subjects in four groups; healthy, gingivitis, and chronic periodontitis (CP) patients with and without SLT use. Saliva samples were collected, and clinical periodontal parameters were recorded at baseline and at one month after non-surgical periodontal therapy. Glutathione levels were analyzed using spectrophotometry at $412 \mathrm{~nm}$. Statistical analysis was carried out using paired t-test, chi-squared, and analysis of variance (ANOVA).

RESULTS Mean glutathione values in saliva were found to be lower in periodontitis patients compared to SLT users at baseline and at 1 month post non-surgical periodontal therapy $(\mathrm{p}<0.001)$ In addition, non-surgical therapy leads to a highly significant improvement in the glutathione levels in gingivitis, in the CP with and without ST groups $(\mathrm{p}<0.001)$.

CONCLUSIONS Successful non-surgical periodontal therapy leads to considerable progress in the redox balance, thus regulating glutathione levels and reducing the effects of SLT on the periodontium. This emphasises the importance of nonsurgical therapy, especially among SLT users.
\end{abstract}

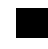

\section{AFFILIATION \\ 1 Department of Periodontics, P.M.N.M. Dental College and Hospital, Bagalkot, India}

\section{CORRESPONDENCE TO \\ Arati C. Koregol. Department of Periodontics, P.M.N.M. Dental College and Hospital, Bagalkot, Karnataka 587103, India. E-mail: aratikperio@yahoo.co.in}

\section{KEYWORDS}

periodontitis, glutathione, reactive oxygen species, antioxidant, smokeless tobacco, non-surgical periodontal therapy

Received: 6 June 2019

Revised: 25 November 2019

Accepted: 4 December 2019

\section{INTRODUCTION}

In normal physiology, equilibrium is maintained between reactive oxygen species (ROS) activity and antioxidant (AO) shielding capacity. When there is an imbalance, caused either by an augmented ROS production or diminution in $\mathrm{AO}$ shielding activity, oxidative stress results. One of the causes of the generation of oxidative stress is tobacco consumption, which generates free radicals ${ }^{1}$.

Free radicals participate in the commencement and evolution of several diseases, including non- insulin dependent diabetes mellitus, cardiovascular disease, metabolic syndrome, acute respiratory distress syndrome, and periodontal disease. Lately, they are thought to be a link between systemic diseases and periodontitis ${ }^{2}$. Increased reactive oxygen species production is exhibited in aggressive and chronic forms of periodontitis due to functionally activated polymorphonuclear leucocytes ${ }^{3}$.

These oxidative products have been detected in various body fluids such as blood, saliva and 
gingival crevicular fluid (GCF). Saliva contains diverse antioxidants that provide almost $70 \%$ of the total radical-trapping antioxidant capacity. Saliva contains non-enzymatic antioxidants such as uric acid, albumin, glutathione, vitamins $\mathrm{A}$ and $\mathrm{C}$, and enzymatics such as superoxide dismutase, glutathione peroxidase, catalase, glutathione, which neutralize free radicals ${ }^{4,5}$. Glutathione $(\mathrm{GSH})$ is a low molecular weight thiol (up to $5-10 \mathrm{mM}$ ) present in the cell, involved as a coenzyme in oxidationreduction reactions in cells. Also, it is a ubiquitous tripeptide made from the combination of three amino acids: cysteine, glutamate, and glycine ${ }^{6}$.

Mechanical debridement has been the cornerstone for professional plaque control and prevention of periodontal disease for centuries. Successful periodontal treatment has been shown to reduce glutathione levels in saliva ${ }^{7}$.

There are few studies evaluating periodontal disease with and without smokeless tobacco and modifications after non-surgical periodontal therapy. Thus, the present study assessed the association between periodontitis, smokeless tobacco and salivary glutathione levels at baseline and at one month after non-surgical periodontal therapy. This study hypothesized that glutathione levels might change after thorough scaling and root planing, which is a procedure that not only preserves periodontal tissues but helps to limit the oral source of inflammation.

\section{METHODS}

\section{Study population}

Subjects, males and females, aged 25-65 years, were randomly selected from the outpatient section of the Department of Periodontics, P.M.N.M. Dental College, Bagalkot, Karnataka, India. The study was approved by the Institutional Ethical Committee.

The power of this study was $80 \%$, at $95 \%$ confidence interval and error margin of $5 \%$, resulting in a required sample size of 100 participants. A written signed informed consent was obtained from the participants who were divided into four groups of 25 members each. Group I was: healthy subjects who had no systemic diseases and showed absence of clinical and radiographic signs of periodontitis, with at least 20 teeth present. Group II was: gingivitis subjects with plaque present at gingival margin, change in colour, contour, and bleeding on probing (BOP) provocation. Group III was: chronic periodontitis subjects with $\mathrm{BOP}, \mathrm{CAL} \geq 3 \mathrm{~mm}$ at $>30 \%$ sites in the mouth, with at least 20 teeth present. Group IV was: chronic periodontitis subjects who were using smokeless tobacco or who reported snuff or chewing tobacco use at least 20 times and who reported using snuff or chewing tobacco at the time of interview ${ }^{8,9}$. Patients of Groups II, III and IV were re-assessed at 1 month after non-surgical periodontal therapy ${ }^{7}$.

The subjects with a history of cigarette smoking, usage of vitamin supplements, history of any antibiotic/anti-inflammatory medication and corticosteroid therapy for 6 months prior to study, any known systemic disease or conditions, pregnant, menopause or lactating women, regular usage of mouthwash, or antioxidants supplement intake during the last 3 months, and periodontal therapy 6 months prior to study, were excluded from the study ${ }^{10}$.

Patient clinical history was recorded in a shortstructured manner for each subject including: demographic data such as age, sex, occupation, and details of smokeless tobacco consumption (duration, type, and frequency), cigarette smoking, medical history, drug history, and dietary supplements such as vitamins, alcohol etc ${ }^{11}$.

\section{Determination of clinical parameters}

Full-mouth periodontal examination was performed by a single examiner after the grouping of the subjects. Periodontal parameters such as gingival index (GI) (Loe and Silness ${ }^{12}$ ), plaque index (PI) (Loe and Silness ${ }^{13}$ ), bleeding on probing (BOP) (Muhlemann and $\mathrm{Son}^{14}$ ), pocket probing depth (PPD) $)^{15}$, and clinical attachment level (CAL), were assessed by a single examiner using a Williams periodontal probe.

\section{Periodontal treatment}

With motivation and instruction in daily plaque control, non-surgical periodontal therapy was performed on patients with gingivitis, and chronic periodontitis with and without smokeless tobacco. Phase I therapy included pre-procedural oral rinse, full-mouth scaling and root planing, along with maintenance and monitoring of oral hygiene. Clinical data and samples were obtained at baseline and at 1 month after non-surgical periodontal therapy ${ }^{7}$. 


\section{Saliva sample collection and analysis}

About $5 \mathrm{~mL}$ of unstimulated saliva was collected from each subject into Eppendorf tubes on the same day. To minimize circadian influences, sample collection was done between 09:00 a.m to 11:00 a.m at least 1 hour after eating or washing of mouth. During collection, subjects were comfortably seated and were instructed to rinse their mouth thoroughly with water. With head tilted slightly down, saliva was allowed to pool in the floor of the mouth for 5 minutes by leaning forward. Subjects were then told to spit into an Eppendorf tube every 60 seconds for 10 minutes or when the subject experienced an urge to swallow the fluid accumulated in the floor of the mouth $^{11,16}$. Samples were centrifuged immediately to remove cell debris $\left(10000 \mathrm{~g} \times 10 \mathrm{~min}\right.$, at $\left.4^{\circ} \mathrm{C}\right)$. The clear supernatants $(50 \mu \mathrm{L}$ each) were gently pipetted out into another Eppendorf tube and stored at $-40^{\circ} \mathrm{C}$ until analyzed. GSH was estimated by the method of Sedlak and Lindsay ${ }^{17}$. Briefly, $5 \%$ saliva was prepared in $20 \mathrm{mM}$ EDTA, $\mathrm{pH} 4.7$ and $100 \mu \mathrm{L}$ of the saliva or pure GSH was added to $0.2 \mathrm{M}$ Tris-EDTA buffer (1.0 mL, pH8.2) and $20 \mathrm{mM}$ EDTA, pH4.7 (0.9 mL) followed by $20 \mu \mathrm{L}$ of Ellman's reagent $(10 \mathrm{mmol} / \mathrm{L}$ DTNB in methanol). After $30 \mathrm{~min}$ of incubation at room temperature, absorbance was read at $412 \mathrm{~nm}$ by spectrophotometer. Samples were centrifuged before the absorbance of the supernatants was measured ${ }^{7,17}$.

\section{Statistical analysis}

Descriptive and bivariate statistics were carried out. The chi-squared test was used to know the gender distribution; the paired t-test was used to find the significance of study parameters on a continuous scale between two groups (inter- and intra-group analysis) on metric parameters. ANOVA was used to find the significance of study parameters between the groups (inter-group analysis) at baseline, and non-parametric Kruskal-Wallis test was used, as part of the data did not follow a normal distribution between the groups (inter-group analysis) at baseline and one month after non-surgical periodontal therapy. Data were expressed as mean and standard deviation (SD). The data collected were analysed using computer software IBM SPSS statistics 20.0.

\section{RESULTS}

A total of 100 subjects were recruited, aged 25-65 years, among which 17 females and 8 males belonged to the healthy group with a mean age of $30.12 \pm 7.59$ years, 15 females and 10 males were in the gingivitis group with a mean age of $33.48 \pm 8.91$ years, 12 females and 13 males in the chronic periodontitis group with mean age of $43.12 \pm 11.61$ years, and 9 females and 16 males were in the dual chronic periodontitis and current smokeless tobacco use with a mean age of $45.04 \pm 11.24$ years. Glutathione levels were found to differ by age but not by gender (data not shown).

The comparison of the clinical parameters and glutathione levels between baseline and follow-up in the different groups' mean clinical parameters are presented in Table 1. Clinical parameters, i.e. gingival index, plaque index, bleeding index, pocket probing depth, clinical attachment loss, and

Table 1. Comparison of the clinical parameters and glutathione levels between baseline and at one month after non-surgical periodontal therapy in the different groups $(\mathrm{N}=25)$

$\begin{array}{lccccc}\text { Variables } & \text { Time point } & \text { Vean } & \text { SD } & \text { P } \\ \text { Gingival index } & \text { A } & 2.208 & 0.2326 & 4.969 & <0.001 \\ \text { Plaque index } & \text { B } & 1.992 & 0.3415 & & <0.001 \\ \text { Bleeding index } & \text { A } & 2.400 & 0.2309 & 7.160 & <0.001 \\ \text { Probing pocket depth } & \text { B } & 2.204 & 0.2458 & & <0.001 \\ \text { Clinical attachment loss } & \text { A } & 2.584 & 0.2322 & 7.365 & <0.001 \\ & \text { B } & 2.332 & 0.2286 & & \end{array}$


Table 1. Continued

\begin{tabular}{|c|c|c|c|c|c|}
\hline Variables & Time point & Vean & SD & $t^{a}$ & $p$ \\
\hline \multirow[t]{2}{*}{ Glutathione } & $\mathrm{A}$ & 3.2344 & 1.04358 & 6.457 & $<0.001$ \\
\hline & B & 5.0112 & 1.10734 & & \\
\hline \multirow[t]{2}{*}{ Gingival index } & $A$ & 2.368 & 0.4571 & 8.642 & $<0.001$ \\
\hline & B & 2.080 & 0.4397 & & \\
\hline \multirow[t]{2}{*}{ Plaque index } & $A$ & 2.468 & 0.4488 & 7.498 & $<0.001$ \\
\hline & B & 2.236 & 0.4545 & & \\
\hline \multirow[t]{2}{*}{ Bleeding index } & $A$ & 2.764 & 0.3902 & 9.397 & $<0.001$ \\
\hline & B & 2.432 & 0.3881 & & \\
\hline \multirow[t]{2}{*}{ Probing pocket depth } & A & 7.64 & 1.121 & 6.722 & $<0.001$ \\
\hline & B & 6.55 & 1.010 & & \\
\hline \multirow[t]{2}{*}{ Clinical attachment loss } & A & 8.16 & 1.070 & 8.971 & $<0.001$ \\
\hline & B & 6.62 & 1.006 & & \\
\hline \multirow[t]{2}{*}{ Glutathione } & A & 2.6532 & 0.81127 & 3.720 & $<0.001$ \\
\hline & B & 3.1296 & 0.79036 & & \\
\hline \multirow[t]{2}{*}{ Gingival index } & $A$ & 1.136 & 0.7325 & 4.637 & $<0.001$ \\
\hline & B & 0.948 & 0.6596 & & \\
\hline \multirow[t]{2}{*}{ Plaque index } & $A$ & 1.348 & 0.6929 & 4.993 & $<0.001$ \\
\hline & B & 1.084 & 0.6196 & & \\
\hline \multirow[t]{2}{*}{ Bleeding index } & $A$ & 1.514 & 0.6969 & 7.771 & $<0.001$ \\
\hline & B & 1.168 & 0.6095 & & \\
\hline \multirow[t]{2}{*}{ Probing pocket depth } & $A$ & 2.32 & 0.476 & 1.549 & 0.134 \\
\hline & B & 2.12 & 0.332 & & \\
\hline \multirow[t]{2}{*}{ Clinical attachment loss } & $A$ & 0 & 0 & NA & NA \\
\hline & B & 0 & 0 & & \\
\hline \multirow[t]{2}{*}{ Glutathione } & $A$ & 3.6512 & 1.02572 & 6.507 & $<0.001$ \\
\hline & B & 4.6388 & 1.12791 & & \\
\hline
\end{tabular}

a Highly significant, paired t-test $p<0.001$. SD: standard deviation. A: At baseline. B: At 1 month.

Table 2. Comparison of the clinical parameters and glutathione levels at baseline among all the groups ( $\mathrm{N}=100)$

\begin{tabular}{|c|c|c|c|c|c|c|}
\hline Variables & Ileallhy & Gingivilis & $C P$ & $\begin{array}{c}\text { CP with } \\
\text { smokeless } \\
\text { tobacco }\end{array}$ & F & $p^{a}$ \\
\hline Gingival index & $0.33 \pm 0.23$ & $1.13 \pm 0.73$ & $2.20 \pm 0.23$ & $2.36 \pm 0.45$ & 106.7 & $<0.001$ \\
\hline Plaque index & $0.37 \pm 0.22$ & $1.34 \pm 0.69$ & $2.4 \pm 0.23$ & $2.46 \pm 0.44$ & 124.67 & $<0.001$ \\
\hline Bleeding index & $0.30 \pm 0.26$ & $1.51 \pm 0.69$ & $2.58 \pm 0.23$ & $2.76 \pm 0.39$ & 169.88 & $<0.001$ \\
\hline Probing pocket depth & $2.0 \pm 0.0$ & $2.32 \pm 0.47$ & $6.82 \pm 0.92$ & $7.64 \pm 1.12$ & 371.21 & $<0.001$ \\
\hline Clinical attachment loss & $0.0 \pm 0.0$ & $0.0 \pm 0.0$ & $7.73 \pm 0.76$ & $8.16 \pm 1.07$ & 1219.90 & $<0.001$ \\
\hline Glutathione & $5.95 \pm 1.40$ & $3.65 \pm 1.02$ & $3.23 \pm 1.04$ & $2.65 \pm 0.81$ & 43.804 & $<0.001$ \\
\hline
\end{tabular}

a Based on ANOVA test. SD: standard deviation. Columns 1-4 give mean \pm SD.

glutathione values in chronic periodontitis patients were found to be significantly different at baseline and at 1 month after non-surgical periodontal therapy, and also among patients with chronic periodontitis that use SLT.

A comparison of the clinical parameters and glutathione levels at baseline among all the groups were found to be significantly different (Table 2), while the comparison of the mean differences of clinical parameters and glutathione levels among all the groups between baseline and at the one month follow-up are presented in Table 3. 
Table 3. Comparison of the mean difference (baseline values minus values at 1 month after non-surgical periodontal therapy) of clinical parameters and glutathione levels among all the groups $(\mathrm{N}=100)$

\begin{tabular}{|c|c|c|c|c|c|}
\hline Variables & Gingivitis & $C P$ & CP SIT & $x^{2}$ & $p^{a}$ \\
\hline GI & $0.18 \pm 0.20$ & $0.21 \pm 0.21$ & $0.28 \pm 0.16$ & 10.136 & 0.006 \\
\hline $\mathrm{PI}$ & $0.26 \pm 0.26$ & $0.19 \pm 0.13$ & $0.23 \pm 0.15$ & 1.358 & 0.507 \\
\hline BOP & $0.34 \pm 0.22$ & $0.25 \pm 0.17$ & $0.33 \pm 0.17$ & 3.530 & 0.171 \\
\hline PPD (mm) & $0.20 \pm 0.64$ & $1.94 \pm 1.17$ & $1.09 \pm 0.81$ & 29.092 & $<0.001$ \\
\hline CAL (mm) & $0.0 \pm 0.0$ & $2.7 \pm 1.1$ & $1.54 \pm 0.85$ & 55.168 & $<0.001$ \\
\hline Glutathione & $0.98 \pm 0.75$ & $1.77 \pm 1.37$ & $0.47 \pm 0.64$ & 18.702 & $<0.001$ \\
\hline
\end{tabular}

a Based on Kruskal-Wallis test. SD: standard deviation. Columns 1-3 give mean \pm SD.

\section{DISCUSSION}

Smokeless tobacco had an effect on clinical parameters of oral health. However, non-surgical periodontal therapy was found to have an impact on this effect. This was expected as oral smokeless tobacco products are placed in the mouth, cheek or lip and sucked or chewed, and so affect oral and periodontal health. Previous research has indicated that the risk of periodontal pockets increases as the duration and tobacco consumption increases ${ }^{18}$.

An immune reaction occurs between pathogenic bacteria and the host body with sub-gingival dental plaque playing a major role for the inflammation within the periodontium. Various factors play critical role in the progression of tissue damage and periodontal disease like interleukin-2,6,8, $\beta$-interferon and TNF-a, MMP, NF-k $\beta$, and AP-1. In turn macrophages, neutrophils and fibroblasts accumulate to generate more ROS, and hence more tissue destruction forming a vicious cycle ${ }^{19,20}$.

Among all markers for periodontal disease, host-derived enzymes in saliva appear to be best indicators as found by Kaufman and Lamster ${ }^{21}$ and later confirmed by Kinney et al. ${ }^{22}$. Although stimulation of saliva flow increases saliva volume, it leads to disruption of the $\mathrm{AO}$ concentration. Hence in the present study, unstimulated saliva samples were collected.

Bleeding on probing can be expected from a patient with inflammation and pocketing, which leads to contamination of saliva samples. In periodontal disease, reduced salivary antioxidant and increased oxidative damage occur, as reported in the study by Sculley and Langley-Evans ${ }^{16}$ where a saliva sample was taken after dental examination.
One factor that affected the study findings is leakage of antioxidants from plasma component into saliva. To avoid this in the current study, all samples were collected before the dental examination ${ }^{11,16}$.

In the present study, glutathione levels showed a positive correlation with age, as found by Lieshout et al. ${ }^{23}$, and was due to detoxification potential, which decreases with age. No gender difference with glutathione levels were seen in the present study, which could be due to no changes in the amount of oxidised glutathione or the activity of glutathione reductase, which can alter the balance between the genetic expression of pro-inflammatory mediators and anti-oxidant enzymes. In contrast, a study by Ongoz et al. ${ }^{7}$ showed a positive correlation between glutathione and gender.

Our study demonstrated that there was a positive association between periodontal clinical parameters, i.e. gingival index, plaque index, bleeding index, probing pocket depth, clinical attachment loss and glutathione levels in gingivitis, chronic periodontitis with and without smokeless tobacco groups after non-surgical periodontal therapy, in accordance with a study conducted by Tinti et al. ${ }^{24}$. This could be due to a decrease in pro-inflammatory cytokine levels after a periodontal intervention.

This study found reduced amounts of salivary glutathione in gingivitis subjects compared to the healthy group, as was also found by Karim et al. ${ }^{25}$ where glutathione was shown to effect gingival fibroblasts. The post-therapy values were found to be significant in the gingivitis group. This, according to an earlier study by Chapple et al. ${ }^{26}$, could be explained by a reduction in bacterial load. The present study identified lower salivary glutathione in 
CP subjects when compared to healthy subjects, and these levels increased after non-surgical periodontal therapy, in accordance with Tsai et al. ${ }^{27}$. In addition, Barnes et al. ${ }^{28}$ reported that increased levels of glutathione in saliva were associated with increased oxidative stress in the patients with periodontal disease. Effect of scaling and root planing on salivary glutathione levels was significant within groups, but not between groups, according to Rai et al. ${ }^{29}$ and Daib et al. ${ }^{30}$. Whereas Tsai et al. ${ }^{27}$ showed positive correlation between groups. The present study further demonstrated that an association between glutathione, periodontitis and smokeless tobacco, could be attributed to increased oxidative stress as a primary etiological feature resulting in release of reactive oxygen species (ROS). Lower salivary glutathione in the chronic periodontitis with smokeless tobacco subjects was shown in the present study when compared to chronic periodontitis, gingivitis and healthy subjects, and these levels increased after non-surgical periodontal therapy, which might be due to synergistic effect of certain pathogens and smokeless tobacco resulting in a pro-inflammatory state, lowering glutathione levels within periodontal cells, and OS depleting glutathione, resulting in turn in activation of redoxsensitive transcription factors ${ }^{20,24}$. Our data indicated that periodontal therapy could improve local changes in the gingival redox balance as well as resolution of periodontal inflammation in $\mathrm{CP}$ with $\mathrm{ST}$ subjects.

\section{Limitations}

The results of this research emphasized the need for studies with a large sample size; so sample size could be a possible limitation of the study. Age and sex matching were not possible, as subjects were recruited for the study as and when they came to the college outpatient department for dental examination.

\section{CONCLUSIONS}

Smokeless tobacco results in an imbalance between AO and ROS, thus this study suggests that periodontitis together with smokeless tobacco lead to significant changes in $\mathrm{AO}$ enzyme activity in saliva. The present study clarifies the possible relationship between anti-oxidant enzyme levels, oxidative stress and tobacco habit, and possible outcomes after non-surgical periodontal therapy. It emphasises the possible importance of AO level determination in unstimulated saliva as a valid and convenient biomarker for periodontal diseases. SLT use coupled with a lesion would probably have a synergistic effect in lowering the body's antioxidant status paving the way for enhanced oxidative stress.

A chairside test for measurement of the effect of smokeless tobacco and salivary glutathione is reliable, easy, and inexpensive. Hence, glutathione may be a valuable tool in periodontal disease diagnosis, prognosis and as a marker of redox status, and in early planning of treatment modalities.

\section{REFERENCES}

1. Patel BP, Rawal UM, Shah PM, Prajapati JA, Rawal RM, Dave TK. Study of tobacco habits and alterations in enzymatic antioxidant system in oral cancer. Oncology. 2005;68(4-6):511-519. doi:10.1159/000086995

2. Bains VK, Bains R. The antioxidant master glutathione and periodontal health. Dent Res J. 2015;12(5):389-405. doi:10.4103/1735-3327.166169

3. Chapple ILC, Brock G, Eftimiadi C, Matthews JB. Glutathione in gingival crevicular fluid and its relation to local antioxidant capacity in periodontal health and disease. J Clin Pathol: Mol Pathol. 2002;55(6):367-373. doi:10.1136/mp.55.6.367

4. Trivedi S, Lal N, Mahdi AA, Singh B, Pandey S. Association of salivary lipid peroxidation levels, antioxidant enzymes, and chronic periodontitis. Int J Periodontics Restorative Dent. 2015;35(2):e14-e19. doi:10.11607/prd.2079

5. Moore S, Calder KA, Miller NJ, Rice Evans CA. Antioxidant activity of saliva and periodontal disease. Free Rad Res. 1994;21(6):417-425. doi:10.3109/10715769409056594

6. Townsend DM, Tew KD, Tapiero H. The importance of glutathione in human disease. Biomed Pharmacother. 2003;57(3-4):145-155. doi:10.1016/s0753-3322(03)00043-x

7. Ongoz Dede F, Bozkurt Dogan S, Balli U, Avci B, Durmuslar MC, Baratzade T. Glutathione levels in plasma, saliva and gingival crevicular fluid after periodontal therapy in obese and normal weight individuals. J Periodont Res. 2016;51(6):726-734. doi:10.1111/jre.12349

8. American Academy Of Periodontology Task Force Report on the Update to the 1999 Classification Of Periodontal Diseases and Conditions. J Periodontol. 2015;86(7):835838. doi:10.1902/jop.2015.157001

9. Use of Smokeless tobacco among adults-United States, 1991. JAMA. 1993;269(23):2971. doi:10.1001/jama.1993.03500230053013

10. Savita M, Sarun E, Arora S, Krishnan S. Evaluation of glutathione level in gingival crevicular fluid in periodontal health, in chronic periodontitis and after nonsurgical periodontal therapy: A clinicobiochemical study. Contemp Clin Dent. 2015;6(2):206-210. doi:10.4103/0976-237x.156047 
11. Kosoko AM, Olayanju OA, Rahamon SK, Arinola OG. Salivary lipid peroxidation and antioxidant status in nigerian cigarette smokers with or without periodontitis. Asian J Med Health. 2017;3(3):1-9. doi:10.9734/ajmah/2017/30749

12. Loe H, Silness J. Periodontal disease in pregnancy. I. Prevalence and severity. Acta Odontol Scand. 1963;21(6):533-551. doi:10.3109/00016356309011240

13. Silness J, Loe H. Periodontal disease in pregnancy. II. Correlation between oral hygiene and periodontal condition. Acta Odontol Scand. 1964;22(1):121-135. doi:10.3109/00016356408993968

14. Muhlemann HR, Son S. Gingival sulcus bleeding - a leading symptom in initial gingivitis. Helv Odontol Acta. 1971;15(2):107-113. PMID:5315729.

15. Russell AL. A system for classification and scoring for prevalence surveys of periodontal disease. J dent Res. 1956;35(3):350-359. doi:10.1177/00220345560350030401

16. Sculley DV and Langley-Evans SC. Periodontal disease is associated with lower antioxidant capacity in whole saliva and evidence of increased protein oxidation. Clin Sci. 2003;105(2):167-172. doi:10.1042/cs20030031

17. Sedlak J, Lindsay RH. "Estimation of total, proteinbound and non-protein sulfhydryl groups in tissue with Ellman's reagent". Anal Biochem. 1968;25:192-205. doi:10.1016/0003-2697(68)90092-4

18. Singh GP, Rizvi I, Gupta V, Bains VK. Influence of smokeless tobacco on periodontal health status in local population of north india: A cross sectional study. Dent Res J. 2011;8(4):211-220. doi:10.4103/1735-3327.86045

19. Dahiya P, Kamal R, Gupta R, Bhardwaj R, Chaudhary $\mathrm{K}$, Kaur S. Reactive oxygen species in periodontitis. J Indian Soc Periodontol. 2013;17(4):411-416. doi:10.4103/0972-124x.118306

20. Borges IJ, Moreira EAM, Filho DW, Oliveira TB, Silva MBS, Frode TS. Proinflammatory and oxidative stress markers in patients with periodontal disease. Mediators Inflamm. 2007;2007:1-5. doi:10.1155/2007/45794

21. Kaufman E, Lamster IB. Analysis of saliva for periodontal diagnosis- A review. J Clin Periodontol 2000;27(7):453465. doi:10.1034/j.1600-051x.2000.027007453.x

22. Kinney JS, Morelli T, Brun T, et al. Saliva/pathogen biomarker signatures and periodontal disease progression. J Dent Res. 2011;90(6):752-758. doi:10.1177/0022034511399908

23. Lieshout E, Peters W. Age and gender dependent levels of glutathione and glutathione s transferase in human lymphocytes. Carcinogenesis. 1998;19(10):1873-1875. doi:10.1093/carcin/19.10.1873

24. Tinti F, Soory M. Mechanisms for redox actions of nicotine and glutathione in cell culture, relevant to periodontitis. Sci Rep. 2012;2:566. doi:10.1038/srep00566

25. Karim S, Pratibha PK, Kamath S, et al. Superoxide dismutase enzyme and thiol antioxidants in gingival crevicular fluid and saliva. Dent Res J (Isfahan). 2012;9(3):266-272. PMID:23087730.
26. Chapple IL. Reactive oxygen species and antioxidants in inflammatory diseases. J Clin Periodontol. 1997;24:287296. doi:10.1111/j.1600-051x.1997.tb00760.x

27. Tsai CG, Chen HS, Chen SL, et al. Lipid peroxidation: a possible role in the induction and progression of chronic periodontitis. J Periodontal Res. 2005;40(5):378-384. doi:10.1111/j.1600-0765.2005.00818.x

28. Barnes VM, Kennedy AD, Panagakos F, et al. Global metabolomic analysis of human saliva and plasma from healthy and diabetic subjects, with and without periodontal disease. PLoS One. 2014;9(8):e105181. doi:10.1371/journal.pone.0105181

29. Rai B, Jain R, Anand S, Kharb S. Total salivary glutathione levels: Periodontitis in smoker and non-smoker. The Internet Journal of Laboratory Medicine. 2008;3(2):4749. doi:10.5580/288d

30. Diab R, Mounayar A, Maalouf E, Chahine R. Beneficial effects of Solanum melongena (Solanaceae) peduncles extracts, in periodontal diseases. J Med Plant Res. 2011;5(11):2309-2315.

\section{ACKNOWLEDGEMENTS}

The authors acknowledge the support of Chandrashekar V M in the biochemical analysis.

\section{CONFLICTS OF INTEREST}

The authors have completed and submitted the ICMJE Form for Disclosure of Potential Conflicts of Interest and none was reported.

\section{FUNDING}

There was no source of funding for this research.

PROVENANCE AND PEER REVIEW

Not commissioned; externally peer reviewed. 